\title{
ANÁLISE DA PRODUÇ̃̃o CIENTÍFICA SOBRE JOGOS ELETRÔNICOS DISPONÍVEIS NOS PORTAIS SCIELO, LILACS E PORTAL DE PERIÓDICOS DA CAPES
}

Recebido em: 30/10/2018

Aceito em: 27/07/2019

Heitor Luiz Furtado ${ }^{1}$

Fundação Universidade Regional de Blumenau

Universidade do Vale do Itajaí

Itajaí - SC - Brasil

Cahuane Corrêa ${ }^{2}$

Universidade Federal do Paraná (UFPR)

Curitiba - PR - Brasil

Michael Cani ${ }^{3}$

Patrick Joshuan Reitz Matendal ${ }^{4}$

Fundação Universidade Regional de Blumenau

Blumenau - SC - Brasil

Marcelo Moraes e Silva ${ }^{5}$

Universidade Federal do Paraná (UFPR)

Curitiba - Paraná - Brasil

\footnotetext{
${ }^{1}$ Doutorando em Educação Física pelo Programa de Pós-Graduação em Educação Física da Universidade Federal do Paraná e Professor no Departamento de Educação Física na Fundação Universidade Regional de Blumenau e Universidade do Vale do Itajaí.

${ }_{2}^{2}$ Mestra em Educação Física pelo Programa de Pós-Graduação em Educação Física da Universidade Federal do Paraná.

${ }^{3}$ Bacharel em Educação Física na Fundação Universidade Regional de Blumenau.

${ }^{4}$ Bacharel em Educação Física na Fundação Universidade Regional de Blumenau.

${ }^{5}$ Doutor em Educação pela Unicamp e Professor Adjunto do Departamento de Educação Física e PósGraduação em Educação Física na Universidade Federal do Paraná.
} 
RESUMO: O presente artigo teve como objetivo analisar a produção científica sobre jogos eletrônicos disponíveis nos portais Scielo, Lilacs e Portal de Periódicos da Capes. Identificando as temáticas, os locais de publicações, o estrato dos periódicos pelo sistema Qualis e os autores e instituições com mais manuscritos. Como resultado foram encontrados 57 artigos em 44 periódicos. As revistas com maior número de publicação são RBCE e Psicopedagógica. Há um leve predomínio de autores com formação em Educação Física, seguidos de Psicologia e Fisioterapia. Destaca-se a heterogeneidade interpretativa do objeto, perpassando por reflexões desde aspectos relacionadas à saúde dos jogadores até reflexões filosóficas sobre o uso dos videogames e dos jogos eletrônicos. As discussões mais presentes são sobre saúde, escola, lazer e comportamentos, o que demonstra uma diversidade epistemológica nas produções científicas.

PALAVRAS CHAVE: Jogos Eletrônicos. Epistemologia. Produção Científica.

\section{ANALYSIS OF SCIENTIFIC PRODUCTION ON ELECTRONIC GAMES AVAILABLE IN PORTALS SCIELO, LILACS AND PORTAL OF PERIODICALS OF CAPES}

ABSTRACT: The present article had as objective to analyze the scientific production on electronic games available in the portals Scielo, Lilacs and Portal of Periodicals of Capes. Identifying the themes, the places of publications, the stratum of journals by the Qualis system and the authors and institutions with more manuscripts. As a result, 57 articles were found in 44 journals. The journals with the highest number of publications are RBCE and Psicopedagógica. There is a slight predominance of authors with degree in Physical Education, followed by Psychology and Physiotherapy. The interpretive heterogeneity of the object is emphasized, ranging from reflections from aspects related to players' health to philosophical reflections on the use of videogames and electronic games. The most present discussions are about health, school, leisure and behaviors, which demonstrates an epistemological diversity in scientific productions.

KEYWORDS: Electronic Games. Knowledge. Scientific Production.

\section{Introdução}

A tecnologia na contemporaneidade, conforme apontam Sibilia (2002), Levy (1999) e Le Breton (2013), está presente em todas as ações do dia-a-dia dos indivíduos.

Negá-la parece um caminho irreversível, restando a necessidade de adaptação e de melhor uso desses instrumentos, sejam eles para fins de divertimento, trabalho e/ou relações sociais. Com a facilidade e a disseminação de acesso à tecnologia, ocorre o surgimento de aparatos eletrônicos, entre eles estão os celulares, televisões, tablets, 
computadores e videogames.

Nesse contexto, por exemplo, a indústria dos jogos eletrônicos desde seu início apresenta constante crescimento. Segundo o site Newzoo (2016), só no Brasil, até o mês de outubro de 2016 o mercado de games movimentou cerca de US\$1,4 bilhão de dólares, conferindo ao país o $11^{\circ}$ lugar no mercado internacional de games, associado também aos 59,2 milhões de jogadores de videogames existentes em todo o país.

Azevedo (2004) e Jensen; Caprano; Moraes e Silva (2017), lembram que os jogos eletrônicos ocupam parte significativa na vida de milhões de indivíduos, superando o entendimento de ser apenas prática de diversão, sendo inclusive possibilidade de trabalho para muitos, indivíduos como: produtores, designers, e até mesmo novos jogadores profissionais que não só no Brasil, mas no mundo todo estão se tornando cada vez mais conhecidos e valorizados.

Os E-sports (esportes eletrônicos) ainda são fenômenos recentes e causam certos estranhamentos em alguns indivíduos, porém, seu crescimento tem sido substancial, conforme salienta Jensen; Caprano; Moraes e Silva (2017). Em 2015, segundo o site Newzoo (2016), por exemplo, a soma de todos os torneios de E-sports rendeu cerca de US\$61 milhões em premiações, e já possui cerca de 148 milhões de aficionados, inclusive ocupando lugar destacado na programação de canais televisivos abertos.

Com o avanço significativo das tecnologias e a popularização dos jogos eletrônicos apontados anteriormente, surge um novo campo de intervenção e principalmente de produções científicas para a esfera acadêmica/científica. Discutir, compreender, analisar os jogos eletrônicos e suas relações com o campo científico são pontos importantes na medida em que podem fornecer maiores indícios que auxiliam no entendimento desse fenômeno social. Analisar a produção científica sobre determinado 
objeto, nesse caso os jogos eletrônicos, pode fornecer aos interessados maiores indícios do que tem se produzido, quais temáticas são mais exploradas e com isso apresentar peculiaridades a serem levantadas, investigadas e analisadas pelo universo científico.

Nesse sentido, o presente artigo busca responder as seguintes questões: De que maneira se repercutem na publicação de artigos acadêmico-científicos os jogos eletrônicos? O que tem sido produzido acerca desse objeto? Quais temáticas são mais privilegiadas e/ou negligenciadas? Sendo assim, o texto tem como objetivo mapear uma parte da literatura científica, no que tange a publicação em periódicos indexados nas bases de dados: Scielo, Lilacs e Portal de Periódicos da Capes, identificando as temáticas mais atendidas, os locais de publicação, os estratos dos periódicos publicados no sistema Qualis, bem como os autores e instituições com maior número de artigos publicados.

\section{Metodologia}

O presente estudo caracteriza-se como exploratório e descritivo, pois foi realizado um mapeamento da produção científica sobre os jogos eletrônicos disponível em periódicos brasileiros. Elegeu-se para compor o escopo os artigos indexados nas seguintes bases de dados: Scielo, Lilacs e Portal de Periódicos da Capes ${ }^{6}$.

As buscas foram realizadas com a utilização do descritor: "Jogos Eletrônicos". O mesmo deveria estar presente em pelo menos um dos seguintes elementos: título, palavras-chave ou resumo. Após a coleta, foram selecionados para compor o escopo da pesquisa apenas artigos em periódicos brasileiros online, disponíveis para a leitura, publicados em português e que tivessem recebido revisão por pares. O critério de

\footnotetext{
${ }^{6}$ Coleta de dados realizada em outubro de 2017.
} 
exclusão dos artigos foi o de não haver no título, nas palavras-chave ou no resumo o termo "jogos eletrônicos".

Para os fins do mapeamento, foram catalogadas as seguintes informações nos artigos: a) ano de publicação; b) área de conhecimento; c) revista; d) estratificação no Qualis $^{7}$; e) temática central; f) autores ${ }^{8}$; e g) instituições.

Após as buscas nos bancos de dados foram selecionados para a análise 57 artigos. Para identificar os enfoques temáticos dos trabalhos seguiram-se os seguintes procedimentos: 1) leitura dos resumos e, quando necessário, dos trabalhos completos; 2) listagem dos principais assuntos discutidos pelos artigos; e 3) criação de categorias que dessem conta de agrupar os principais assuntos. Classificaram-se os artigos dentro dos seguintes eixos temáticos elaborados em momento posterior ao processo de catalogação (cada artigo, após a coleta de dados, foi catalogado em apenas um eixo temático):

- Jogos Eletrônicos e Comportamento - influência e possíveis consequências dos jogos eletrônicos no comportamento social, afetivo e psicológico dos indivíduos;

- Jogos Eletrônicos e Corporeidade - relação entre os jogos eletrônicos e suas novas possibilidades de vivências corporais, a partir principalmente de reflexões filosóficas;

- Jogos Eletrônicos e Escola - presença e possibilidade de utilização dos jogos eletrônicos como ferramentas nos processos de ensino aprendizagem na escola e demais espaços educativos;

- Jogos Eletrônicos e Esporte - jogos eletrônicos como manifestações

\footnotetext{
${ }^{7}$ Para fins de análise foi considerado o maior Qualis do periódico, independentemente da área.

${ }^{8}$ Para compor as informações dos autores com maior número de publicações e suas respectivas instituições, foi utilizada a plataforma do Currículo Lattes.
} 
esportivas, incluindo discussões acerca do processo de esportivização e do surgimento de atletas;

- Jogos Eletrônicos e Saúde - influência dos jogos eletrônicos na saúde dos indivíduos apontando para possíveis patologias;

- Jogos Eletrônicos, Lazer e Sociedade - relação entre os jogos eletrônicos e as novas possibilidades de lazer contemporâneo e suas repercussões na sociedade.

A criação e catalogação dos autores nos eixos temáticos citados anteriormente, foram por vezes problemáticas, na medida em que vários trabalhos poderiam ser enquadrados em um ou mais eixos. Porém, mesmo sabendo dessa limitação optou-se por tal classificação.

Para a exposição dos resultados da pesquisa, inicialmente apresenta-se os números gerais - indicando a quantidade de publicações sobre os jogos eletrônicos referentes a cada enfoque temático. Na sequência, detalha-se os periódicos e seus respectivos Qualis e, posteriormente, apresenta-se os autores e instituições com maior produtividade e os assuntos abordados em cada um dos eixos.

\section{Resultados e Discussões}

De forma inicial, torna-se necessário visualizar a distribuição dos artigos ano a ano, conforme apresenta o Gráfico 1: 


\section{Gráfico 1: Distribuição da produção por ano - Sistematizado pelos autores.}

\section{Anos de Publicação}

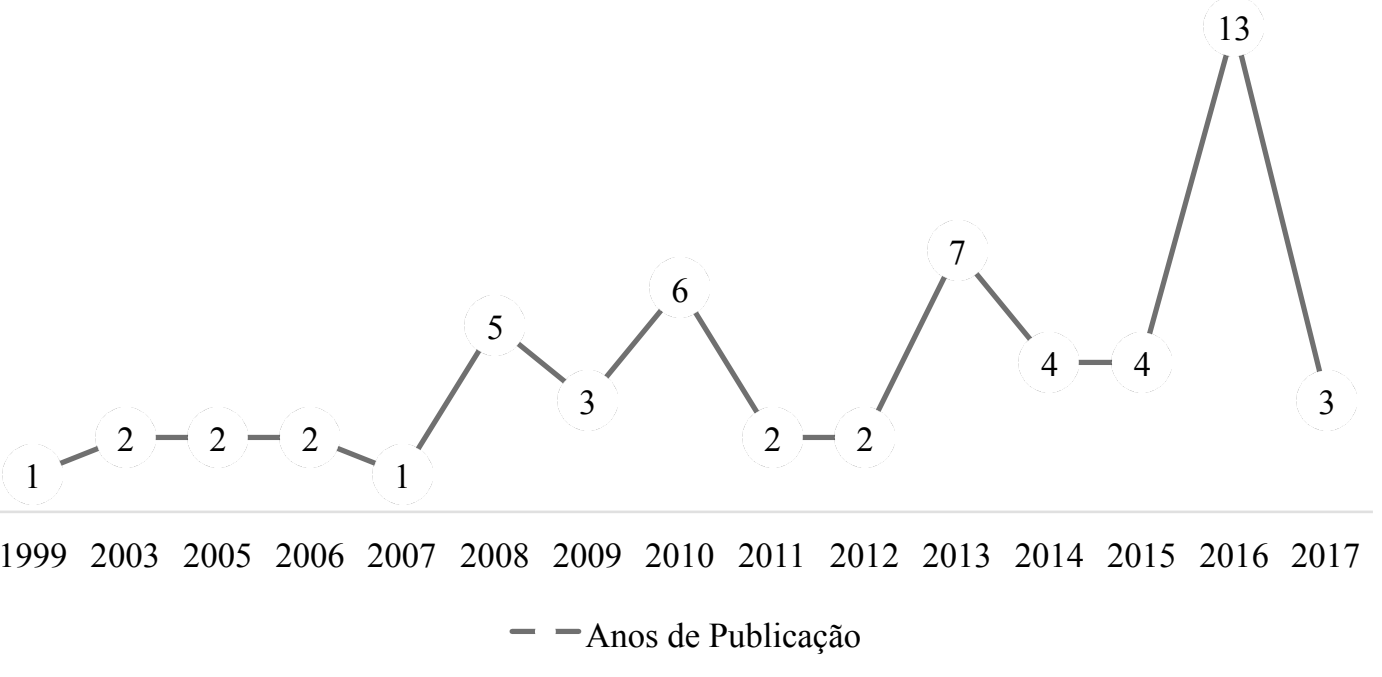

O Gráfico 1 representa a distribuição dos 57 artigos selecionados. Percebe-se maior quantidade de produções no ano de 2016, com 13 manuscritos - o que representa $22,80 \%$ do total - seguidos dos anos de 2013, com sete trabalhos (12,28\%), 2010 com seis pesquisas $(10,52 \%)$, e ainda 2008 , com cinco artigos $(8,77 \%)$. Nos demais anos percebe-se produção tímida oscilando entre uma, duas ou três produções, exceto nos anos de 2014 e 2015, com quatro textos cada. Os dados apontam que não é possível identificar uma distribuição lógica e uniforme, nem demonstrar crescimento nos números absolutos das publicações.

Em relação à distribuição por estratos de avaliação no Qualis (CAPES), os artigos foram classificados apenas pelas melhores avaliações no sistema. Tal escolha justifica-se pelo fato de que as produções estão disponíveis em periódicos científicos situados em diferentes áreas avaliativas. O gráfico a seguir apresenta a classificação dos artigos quanto aos estratos das revistas. 


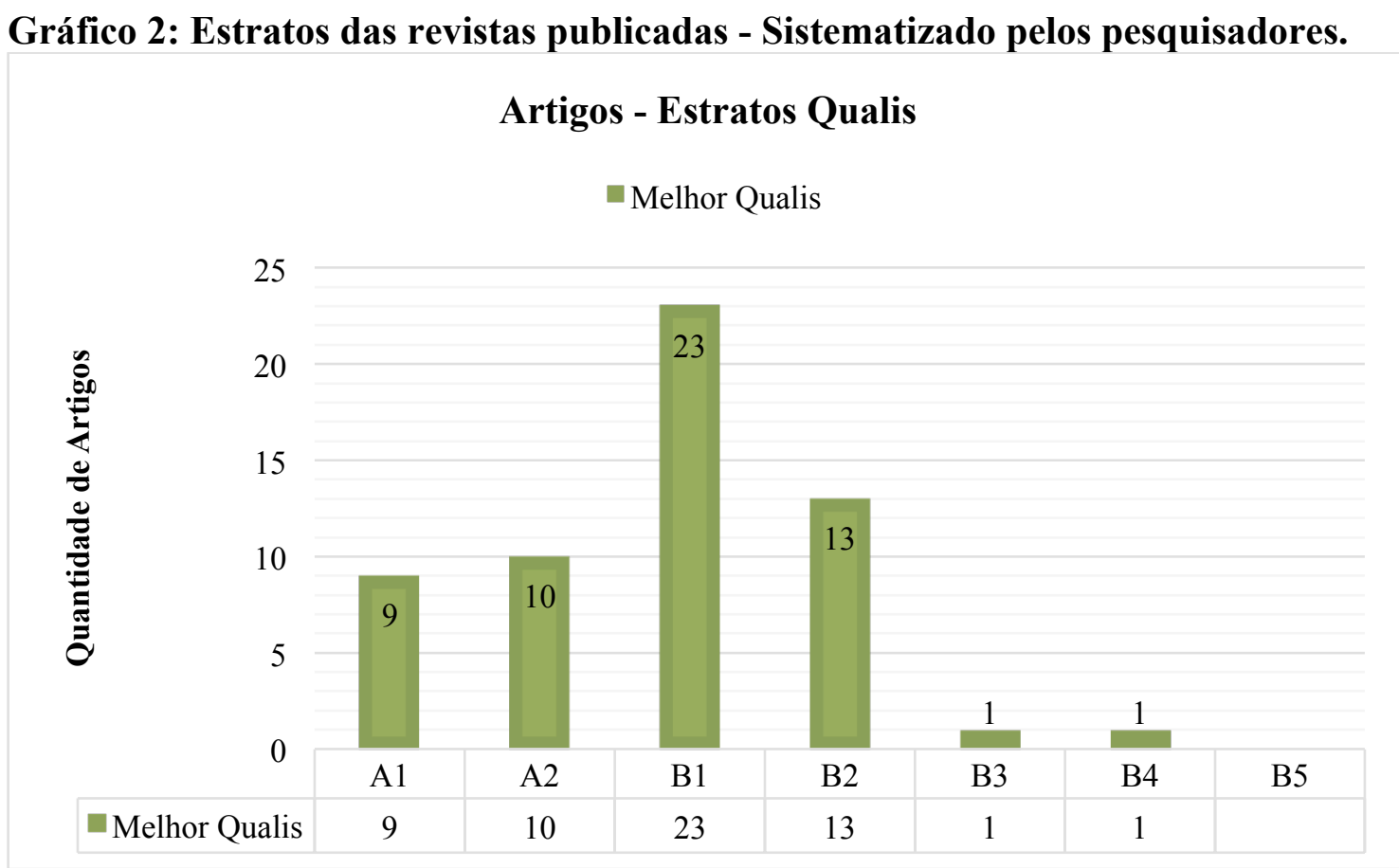

Os artigos publicados em periódicos com estrato B1 representam maior número, contabilizando um total de 23 artigos (40,35\%). O estrato B2 soma 13 textos, o que representa 22,80\% das produções. Algo importante a ser destacado é o lugar ocupado pelas publicações em revista com os melhores estratos avaliativos. Periódicos A1 e A2, somaram 15,78\% e 17,54\%, respectivamente. Além disso, revistas B3 e B4 representaram $1,75 \%$ cada. Nesse sentido, os achados indicam que quase a totalidade das publicações acerca dos jogos eletrônicos situa-se em periódicos classificados nos estratos superiores e medianos, localizados em classificações entre A1 e B2, o que pode indicar uma boa qualidade das publicações, uma vez que se encontram em periódicos com boa avaliação.

Quanto aos periódicos, foram encontradas 44 diferentes revistas. Dos 57 manuscritos publicados, 14 trabalhos estão em periódicos relacionados à área da Psicologia, o que representa $24,56 \%$ das produções sobre os jogos eletrônicos. Em revistas da área da Educação Física foram publicados 11 artigos (21,05\%), seguidos de 
seis trabalhos da área da Educação (10,52\%) e cinco artigos da área da Medicina (8,77\%). Aproximadamente $35 \%$ dos textos estão publicados em periódicos de diferentes áreas, algumas de caráter multidisciplinar, outras de caráter mais específico como, por exemplo, Saúde Coletiva, Filosofia, Fisioterapia, Informática, Lazer etc.

Esses dados apontam para uma heterogeneidade interpretativa advinda de diferentes matrizes científicas. Percebe-se um predomínio, mesmo que sutil, de discussões acerca dos jogos eletrônicos em análises situadas em referenciais teóricos, metodológicos e epistemológicos da Psicologia, seguidas de análises do campo acadêmico/científico da Educação Física. Esses dados apontam para uma multiplicidade interpretativa acerca do fenômeno, perpassando análises alicerçadas em diferentes áreas do conhecimento.

Afim de uma melhor interpretação, a Tabela abaixo apresenta as sete revistas científicas com as maiores quantidades de artigos sobre jogos eletrônicos, e seus respectivos escopos.

Tabela 1: Revistas que mais publicaram sobre jogos eletrônicos, seus respetivos escopos e Qualis Avaliativos'.

\begin{tabular}{|c|c|c|c|c|}
\hline Revistas & Escopo da Revista & $\begin{array}{l}\text { Maior } \\
\text { Qualis }\end{array}$ & $\begin{array}{l}\text { Número de } \\
\text { Artigos }\end{array}$ & $\%$ \\
\hline $\begin{array}{l}\text { Revista Brasileira de } \\
\text { Ciências do Esporte }\end{array}$ & $\begin{array}{l}\text { Publica prioritariamente } \\
\text { artigos originais sobre os } \\
\text { diversos temas } \\
\text { relacionados à Educação } \\
\text { Física/Ciências do } \\
\text { Esporte }\end{array}$ & B1 & 5 & 8,77 \\
\hline Revista Psicopedagogia & $\begin{array}{l}\text { Publica artigos inéditos } \\
\text { na área de } \\
\text { psicopedagogia, em } \\
\text { especial: resultados de } \\
\text { pesquisa de caráter } \\
\text { teórico/empírico; revisões } \\
\text { críticas da literatura de }\end{array}$ & B2 & 3 & 5,26 \\
\hline
\end{tabular}

\footnotetext{
${ }^{9}$ As informações contidas na Tabela 1 foram encontradas nos portais eletrônicos de cada periódico.
} 
Heitor Luiz Furtado, Cahuane Corrêa,

Michael Cani, Patrick Joshuan Reitz Matendal e

Marcelo Moraes e Silva

\begin{tabular}{|c|c|c|c|c|}
\hline & $\begin{array}{l}\text { pesquisa educacional, } \\
\text { temática ou metodológica } \\
\text { e reflexões críticas sobre } \\
\text { experiências pedagógicas }\end{array}$ & & & \\
\hline $\begin{array}{l}\text { Revista Ciência e } \\
\text { Saúde Coletiva }\end{array}$ & $\begin{array}{c}\text { Editada pela Associação } \\
\text { Brasileira de Saúde } \\
\text { Coletiva, é um espaço } \\
\text { científico para discussões, } \\
\text { debates, apresentação de } \\
\text { pesquisas, exposição de } \\
\text { novas ideias e de } \\
\text { controvérsias sobre a área } \\
\text { de caráter multidisciplinar }\end{array}$ & A1 & 2 & 3,50 \\
\hline Ciências \& Cognição & $\begin{array}{l}\text { Estudo da cognição e seus } \\
\text { processos, a partir do } \\
\text { olhar interdisciplinar, } \\
\text { estabelecendo um diálogo } \\
\text { acadêmico entre as } \\
\text { chamadas Ciências } \\
\text { Cognitivas }\end{array}$ & B1 & 2 & 3,50 \\
\hline $\begin{array}{c}\text { Jornal Brasileiro de } \\
\text { Psiquiatria }\end{array}$ & $\begin{array}{l}\text { Publicar estudos com } \\
\text { objetivo de avanço do } \\
\text { conhecimento sobre os } \\
\text { transtornos mentais e a } \\
\text { melhoria da assistência e } \\
\text { cuidado dos pacientes que } \\
\text { sofrem dessas condições }\end{array}$ & B1 & 2 & 3,50 \\
\hline $\begin{array}{c}\text { Psicologia: teoria e } \\
\text { prática }\end{array}$ & $\begin{array}{c}\text { Publicar trabalhos } \\
\text { inéditos em Psicologia e } \\
\text { suas interfaces com áreas } \\
\text { afins }\end{array}$ & A2 & 2 & 3,50 \\
\hline $\begin{array}{c}\text { Revista Paulista de } \\
\text { Pediatria }\end{array}$ & $\begin{array}{l}\text { Publica estudos de casos } \\
\text { clínicos de investigação } \\
\text { metodológica com } \\
\text { abordagem na área da } \\
\text { saúde e pesquisa de } \\
\text { doenças dos recém- } \\
\text { nascidos, lactantes, } \\
\text { crianças e adolescentes }\end{array}$ & B1 & 2 & 3,50 \\
\hline
\end{tabular}

A revista com o maior número de artigos publicados é a Revista Brasileira de Ciências do Esporte, com cinco manuscritos. Destacam-se ainda os seguintes periódicos: Revista Psicopedagogia, Psicologia: teoria e prática e Ciências e Cognição, com predomínio de questões relativas à Psicologia. A Revista Paulista de Pediatria e o 
Jornal Brasileiro de Psiquiatria sugerem enfoques analíticos a partir de referenciais teóricos da Medicina, podendo ainda ser enquadrada a Revista Ciência e Saúde Coletiva que, mesmo tendo caráter multidisciplinar, possui forte tendência a estudos vinculados à área da Saúde.

No que diz respeito aos escopos das revistas com os maiores números de publicações, existem aquelas cujos focos se direcionam a especificidades temáticas como, por exemplo, a Revista Paulista de Pediatria e o Jornal Brasileiro de Psiquiatria, e outras de caráter mais abrangente como a Revista Brasileira de Ciências do Esporte e a Revista Ciência e Saúde Coletiva. Como já mencionado anteriormente, a partir dos estratos avaliativos, as revistas com maiores números de trabalhos publicados também apontam para a diversidade epistemológica no trato com o objeto, perpassando análises interpretativas situadas tanto em referências teórico-metodológicas das ciências naturais, como das ciências humanas e sociais.

Nos 57 artigos encontrados, foram identificados 142 autores, oriundos de diferentes áreas de formação. O gráfico a seguir apresenta os autores e suas respectivas áreas de formação. 
Gráfico 3: Graduação dos autores que produziram sobre jogos eletrônicos Sistematizado pelos autores.

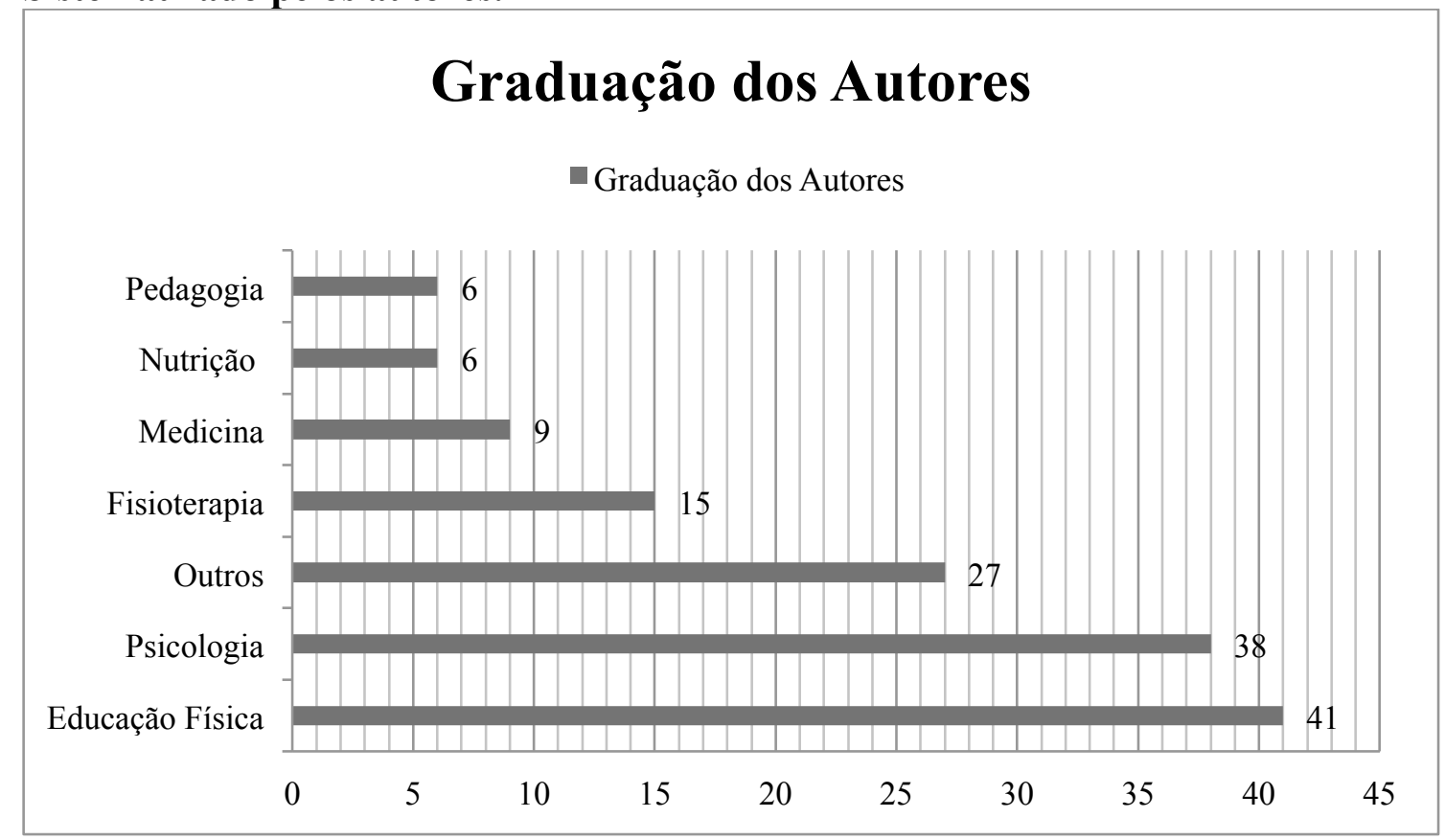

Quanto a área de formação inicial existe um leve predomínio de pesquisadores da área da Educação Física (29,07\%), seguidos de investigadores com formação em Psicologia (26,95\%). Aqueles com formação em Fisioterapia representam 10,63\% do total, já os da área de Medicina, Nutrição e Pedagogia representam 6,38\%, 4,25\% e $4,25 \%$ respectivamente. Tais dados indicam que $77,29 \%$ dos autores estão situados na grande área da Saúde, conforme classificação sugerida pela Coordenadoria de Aperfeiçoamento Pessoal de Nível Superior (CAPES). Outras formações iniciais como Matemática, Odontologia, Farmácia, Ciência Sociais e Ciências da Computação representam uma porcentagem significativa de $19,14 \%$ dos autores. Como já mencionado, as formações iniciais dos autores apontam para uma diversidade de olhares acerca dos jogos eletrônicos, salientando ainda que, cada área possui processos internos de produção do conhecimento distintos, que se traduzem em diferentes formas de se fazer ciência. 
Dos 144 autores encontrados, 82 possuem formação em nível de doutorado $(58,15 \%), 13$ estão em processo de doutoramento $(9,21 \%), 12$ são mestres $(8,51 \%), 3$ são mestrandos (2,12\%), 6 autores possuem especialização (4,25\%) e 13 possuem apenas graduação $(9,21 \%)$. Salienta-se que tais titulações estão em diferentes áreas do conhecimento e foram realizadas em distintas instituições e regiões do país. Como existe um predomínio substancial de autores com a titulação de doutorado pode-se apontar certo amadurecimento na produção dos manuscritos, tendo em vista que se trata da última titulação acadêmica de um pesquisador.

No decorrer da investigação pode-se observar que alguns autores apareceram com maior frequência nas produções. O Quadro a seguir apresenta os autores com mais publicações sobre jogos eletrônicos.

Tabela 2: Autores, instituições e titulação que mais publicaram - Sistematizado pelos pesquisadores.

\begin{tabular}{|c|c|c|c|c|c|c|c|}
\hline Autor & $\begin{array}{c}\mathrm{N}^{\mathrm{o}} \text { de } \\
\text { trabalhos }\end{array}$ & Graduação & $\begin{array}{c}\text { Última } \\
\text { Titulação }\end{array}$ & $\begin{array}{c}\text { Instituição } \\
\text { de } \\
\text { Titulação }\end{array}$ & $\begin{array}{c}\text { Instituição } \\
\text { Atual }\end{array}$ & $\begin{array}{l}\text { Co- } \\
\text { autorias }\end{array}$ & $\begin{array}{l}\text { Relação de } \\
\text { Orientação }\end{array}$ \\
\hline $\begin{array}{c}\text { 1-Claudia Broetto } \\
\text { Rosetti }\end{array}$ & 3 & Psicologia & Doutora & USP & UFES & 13 & $\begin{array}{c}\text { Orientou } \\
13\end{array}$ \\
\hline $\begin{array}{l}\text { 2- Daniela Karine } \\
\text { Ramos }\end{array}$ & 3 & $\begin{array}{l}\text { Psicologia/ } \\
\text { Pedagogia }\end{array}$ & Doutora & UFSC & UFSC & $\mathrm{X}$ & $\mathrm{X}$ \\
\hline $\begin{array}{c}\text { 3- Michelle } \\
\text { Katherine } \\
\text { Andrade Xavier }\end{array}$ & 3 & Fisioterapia & Mestre & UPE & UPE & $4-5-11-14$ & $\begin{array}{l}\text { Orientada } \\
\text { por } 4\end{array}$ \\
\hline $\begin{array}{l}\text { 4- Rodrigo } \\
\text { Cappato de } \\
\text { Araújo }\end{array}$ & 3 & Fisioterapia & Doutor & UFMG & UPE & $3-5-11-14$ & $\begin{array}{c}\text { Orientou } 3 \\
10 \text { e } 14\end{array}$ \\
\hline $\begin{array}{l}\text { 5- Ana Carolina } \\
\text { Rodarti Pitangui }\end{array}$ & 2 & Fisioterapia & Doutor & USP & UPE & $3-4-11-14$ & $\mathrm{X}$ \\
\hline 6- Carlos Baum & 2 & Psicologia & Doutor & UFRGS & UFRGS & $5-6$ & $\begin{array}{l}\text { Orientado } \\
\text { por } 7\end{array}$ \\
\hline $\begin{array}{c}\text { 7- Cleci } \\
\text { Maraschin }\end{array}$ & 2 & Psicologia & Doutor & UFRGS & UFRGS & $5-6$ & Orientou 6 \\
\hline
\end{tabular}


Heitor Luiz Furtado, Cahuane Corrêa, Michael Cani, Patrick Joshuan Reitz Matendal e Marcelo Moraes e Silva

\begin{tabular}{|c|c|c|c|c|c|c|c|}
\hline $\begin{array}{l}\text { 8- Daniel Tornaim } \\
\text { Spritzer }\end{array}$ & 2 & Medicina & Mestre & UFRGS & UFRGS & $\mathrm{X}$ & $\mathrm{X}$ \\
\hline $\begin{array}{c}\text { 9- Fernando } \\
\text { Renato Cavichiolli }\end{array}$ & 2 & $\begin{array}{l}\text { Educação } \\
\text { Física }\end{array}$ & Doutor & UNIMEP & UFPR & 13 & $\begin{array}{l}\text { Orientou } \\
12\end{array}$ \\
\hline $\begin{array}{l}\text { 10- Georgia } \\
\text { Rodrigues Reis } \\
\text { Silva }\end{array}$ & 2 & Fisioterapia & Doutoranda & $\begin{array}{c}\text { Fundação } \\
\text { Oswaldo } \\
\text { Cruz }\end{array}$ & UPE & $3-4-5-14$ & $\begin{array}{l}\text { Orientada } \\
\text { por } 4\end{array}$ \\
\hline $\begin{array}{l}\text { 11- Igor Lins } \\
\text { Lemos }\end{array}$ & 2 & Psicologia & Doutor & UFPE & UFPE & $X$ & $X$ \\
\hline $\begin{array}{l}\text { 12- Leoncio José } \\
\text { de Almeida Reis }\end{array}$ & 2 & $\begin{array}{l}\text { Educação } \\
\text { Física }\end{array}$ & Doutor & UFPR & UFPR & 10 & $\begin{array}{l}\text { Orientado } \\
\text { por } 9\end{array}$ \\
\hline $\begin{array}{c}\text { 13- Maria Thereza } \\
\text { Costa Coelho de } \\
\text { Souza }\end{array}$ & 2 & Psicologia & Doutora & USP & USP & $1-13$ & $\begin{array}{l}\text { Orientada } \\
\text { por } 1\end{array}$ \\
\hline $\begin{array}{c}\text { 14- Valéria } \\
\text { Mayaly Alves de } \\
\text { Oliveira }\end{array}$ & 2 & Fisioterapia & Doutoranda & UPE & UPE & $3-4-5-11$ & $\begin{array}{l}\text { Orientada } \\
\text { por } 4\end{array}$ \\
\hline
\end{tabular}

Claudia Broetto Rosetti, Daniela Karine Ramos, Michelle Katherine Andrade Xavier e Rodrigo Cappato de Araújo publicaram três estudos cada, sendo os pesquisadores com maior número de artigos. Rosetti possui graduação em Psicologia pela Universidade Federal do Espírito Santo (UFES), mestrado em psicologia pela mesma instituição e doutorado em Psicologia Escolar e do Desenvolvimento Humano pela Universidade de São Paulo (USP). Atualmente é docente do Departamento de Psicologia da UFES e faz parte do Programa de Pós-Graduação pela mesma instituição. Seus estudos são voltados aos aspectos afetivos e cognitivos presentes na prática de jogos e brincadeiras. Já Daniela Karine Ramos possui graduação em Psicologia e Pedagogia, mestrado e doutorado na área da Educação pela Universidade Federal de Santa Catarina (UFSC). Exerce docência no Departamento de Metodologia de Ensino e do Programa de Pós-Graduação em Educação da UFSC. Seus estudos focam na educação à distância, uso das tecnologias na educação, jogos eletrônicos e nos aspectos didáticos do processo de ensino aprendizagem. 
Xavier por sua vez, possui graduação em Fisioterapia pela Universidade Estadual da Paraíba (UEPE) e mestrado em Hebiatria pela Universidade Federal de Pernambuco (UFPE). É também docente auxiliar na UPB e fisioterapeuta no Hospital Geral Clériston Andrade. Seus estudos são voltados a avaliação, prescrição e tratamento de dores de origem musculoesqueléticas em adolescentes. Já Araújo possui graduação em Fisioterapia pela Universidade de Ribeirão Preto, mestrado em Medicina pela USP e doutorado em Bioengenharia pela Universidade Federal de Minas Gerais (UFMG). Atualmente é docente no Programa Associado de Pós-Graduação em Educação Física da UPE/UFPB. Seus estudos situam-se na biomecânica, avaliação da dor, programas de treinamento e reabilitação funcional.

Dos 14 autores com os maiores números de artigos publicados, seis possuem graduação em Psicologia, cinco em Fisioterapia, dois em Educação Física e um em medicina. Além disso, com exceção de Daniela Karine Ramos - que de três artigos, escreveu dois individualmente - os autores apresentaram redes de produção de artigos em coautorias.

Em relação ao número de autores, nos 57 artigos publicados verifica-se que foram encontrados 10 artigos (17,54\%) com apenas um autor e 21 textos com 2 autores, o que corresponde a $36,84 \%$ do total. Apenas 7 artigos (12,28\%) continham três autores. Com quatro, cinco e seis autores, o total foi de $10(17,54 \%), 3(5,26 \%)$ e $4(7,01 \%)$ artigos, respectivamente. E para finalizar, apenas uma publicação continha mais de 6 autores, o que contabiliza $1,75 \%$ do montante total. Dos 14 autores com os maiores números de trabalhos publicados, apenas três (Daniela Karine Ramos, Igor Lins Lemos e Daniel Tornaim Spritzer) não possuem trabalhos feitos colaborativamente entre si.

Os dados apontam uma tendência de colaboração entre os autores na elaboração 
dos trabalhos, algo já salientado por Lazarotti Filho et al (2012). Da mesma forma, os achados empíricos se assemelham aos resultados de Gonçalves et al. (2017) sobre a modalidade Basquetebol; e de Moreira et al. (2017) sobre o Voleibol. Balancieri et al. (2005) salientam que os estudos desenvolvidos em coautorias caracterizam o fazer científico contemporâneo e podem auxiliar na construção dos trabalhos, que culminam em se tornar mais bem desenvolvidos e com maiores potenciais de publicação. $\mathrm{O}$ sistema de coautoria parece ter sua sustentação associada a relações de orientador e orientando, visto que dos 14 autores com os maiores números de publicações, 10 deles possuem essa relação.

Dos 144 autores encontrados nos diferentes artigos, foram identificadas 41 diferentes instituições de vínculos. Destaca-se a Universidade Federal de Pernambuco (UFPE) e a Universidade Federal de Santa Catarina (UFSC), ambas com 14 pesquisadores vinculados, e ainda, a Universidade de São Paulo (USP) com 12 pesquisadores. Já a Universidade Federal de Pelotas, a Universidade Federal do Espírito Santo e a Universidade Federal do Paraná possuem apenas cinco pesquisadores vinculados ao tema.

A diversidade de periódicos de autores de distintas áreas de formação e com diferentes titulações - que publicaram estudos relacionados aos jogos eletrônicos indicam que as pesquisas foram realizadas em diferentes eixos e perspectivas interpretativas. Essa heterogeneidade pode ser visualizada no Gráfico 4: 


\section{Gráfico 4: Distribuição de artigos por eixos temáticos - Sistematizado pelos autores.}

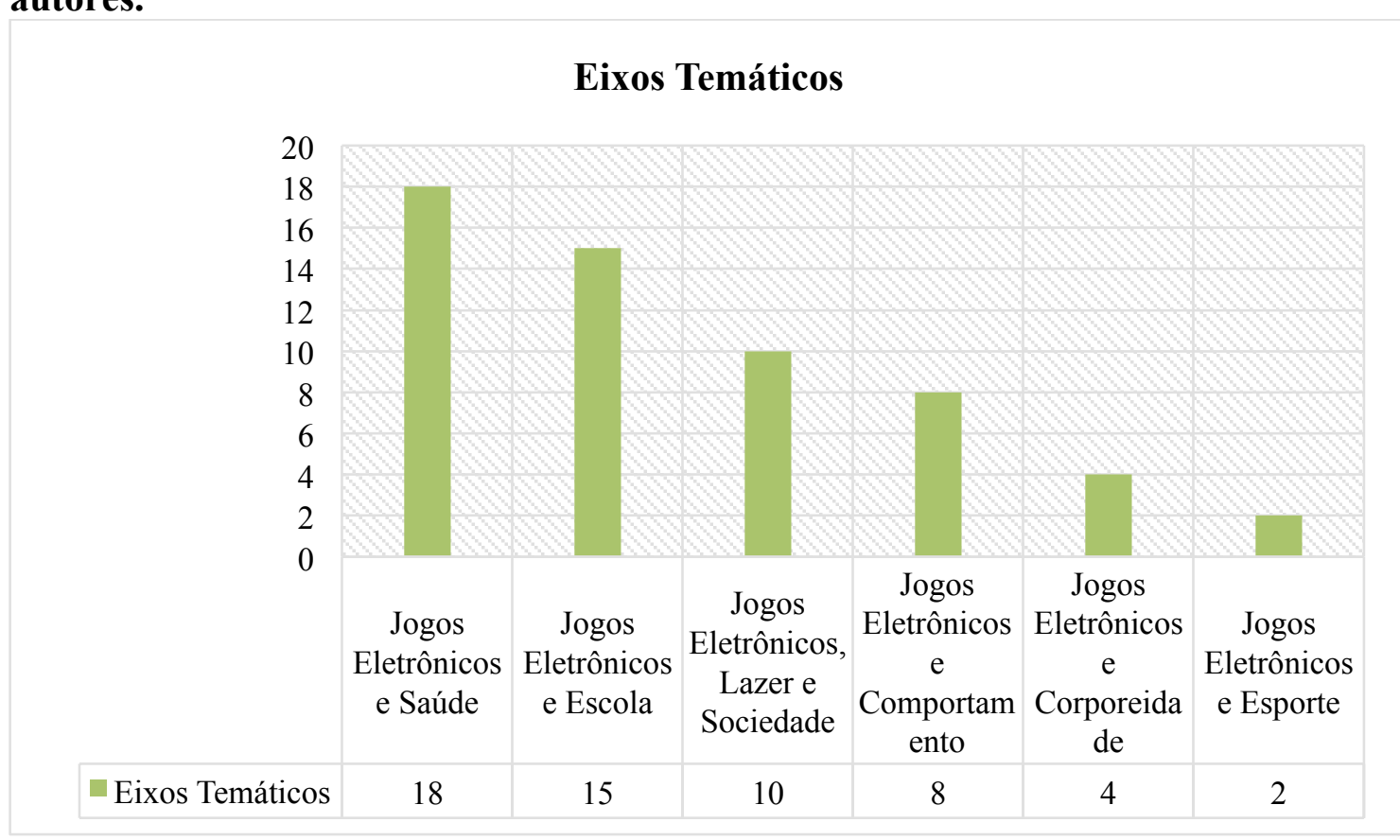

Os artigos enquadrados no eixo temático Jogos Eletrônicos e Saúde são predominantes, com 18 publicações, o que corresponde a 31,57\% dos trabalhos publicados. O de Jogos Eletrônicos e Escola ocupa o segundo lugar no número de publicações com 15 artigos (26,31\%), seguido por Jogos Eletrônicos, Lazer e Sociedade com 10 produções $(17,54 \%)$ Já os eixos temáticos Jogos Eletrônicos e Comportamento, Jogos Eletrônicos e Corporeidade e Jogos Eletrônicos e Esporte, correspondem a $8(14,03 \%), 4(7,01 \%)$ e $2(3,50 \%)$ publicações respectivamente.

Com o maior número de trabalhos, o eixo temático "Jogos Eletrônicos e Saúde" inclui artigos que analisaram os jogos eletrônicos a partir de referências teóricas das ciências biológicas. Seu predomínio possui fortes relações com o percentual de $74 \%$ dos autores que têm formação inicial na área da saúde. Os trabalhos nesse eixo buscaram analisar possíveis consequências, danos à saúde dos indivíduos, tais como: obesidade, problemas decorrentes da inatividade física e possíveis patologias. O trabalho de 
Figueiredo e Sbissa (2013), por exemplo, investigou o efeito de jogos eletrônicos sobre a atenção seletiva em crianças. Os resultados encontrados nesse artigo apontam que a prática de jogos eletrônicos pode influenciar a habilidade cognitiva, alterando a capacidade de aprendizado dos indivíduos. Outro trabalho situado nesse eixo foi o de Ferrari, et al. (2015), no qual analisou a atividade física moderada e vigorosa (AFMV) e o índice de massa corporal (IMC) em crianças que tinham TV's, computadores e jogos de vídeos no quarto. Das crianças selecionadas para o estudo, 45,3\% tinham excesso de peso/obesidade, meninas com computador no quarto (45 min/dia) faziam menos AFMV do que as que não tinham $(51,4 \mathrm{~min} / \mathrm{dia})$, valores semelhantes se fizeram presentes também nos meninos. Para os autores, equipamentos eletrônicos no quarto das crianças podem afetar negativamente a AFMV e o IMC independentemente do sexo, escola e renda familiar anual e contribuir para a inatividade física e obesidade infantil.

No eixo temático "Jogos Eletrônicos e Escola" por sua vez foram encontrados estudos que identificaram a presença e a possibilidade da utilização dos jogos eletrônicos como ferramentas pedagógicas para os processos de ensino aprendizagem nas escolas. Baracho; Grip e Lima (2012), por exemplo, discutiram as perspectivas da utilização da virtualidade dos videogames na educação física escolar, na qual podem ampliar e recriar as possibilidades das práticas e vivências corporais. Para os autores, a cultura digital está cada vez mais inserida no cotidiano, é um caminho inevitável para as atuais e futuras gerações. Salientam ainda que existe a necessidade do professor de Educação Física estar preparado para dialogar com os alunos que vivem nessa nova cultura, sem perder o senso crítico sobre seus usos e considerar uma nova possibilidade de observar e aprender por meio dela. Ao situar tal eixo, o trabalho de Fantin (2015) buscou relacionar os jogos tradicionais, eletrônicos e digitais com os estudos da 
infância, dos jogos e da cultura lúdica. Para a autora, a diversidade que as experiências dos jogos propiciam desafia a escola a construir novas práticas educativas e novos modos de ver a relação entre a tecnologia e as crianças.

No eixo temático "Jogos Eletrônicos, Lazer e Sociedade" os trabalhos versam sobre as possibilidades de interpretação dos jogos eletrônicos como manifestações contemporâneas de lazer e seus possíveis desdobramentos na contemporaneidade. Reis e Cavichiolli (2008), a partir da utilização de referencial teórico de Norbert Elias e Eric Dunning, discutiram a possibilidade de interpretar e analisar os jogos eletrônicos enquanto possibilidades de lazer contemporâneas. Para os autores, os jogos eletrônicos são manifestações do lazer, propiciam excitação e prazer potencializados principalmente pelo comodismo de sua prática. A possibilidade de jogar com outras pessoas via internet é cada vez mais comum, permitindo profundas ressignificações nas convivências sociais contemporâneas; $O$ que resulta, na opinião dos autores, na modificação de hábitos e comportamentos, além de romper com as restrições de práticas de lazer aos ambientes privados e/ou familiares. Ainda nesse eixo, destaca-se o trabalho de Perucia; Balestrin e Verschoore (2011), que analisou a relação entre os jogos eletrônicos e o mercado consumidor, buscando compreender como as empresas que desenvolvem jogos eletrônicos e coordenam suas atividades de produção na indústria brasileira de jogos eletrônicos. Os resultados encontrados apontam um crescimento da importância econômica dos jogos eletrônicos, porém no Brasil o mercado ainda é recente e caracterizado por problemas estruturais, tais como: baixa renda da população, exclusão digital e pirataria.

Os artigos classificados no eixo "Jogos Eletrônicos e Comportamento" apresentam estudos com foco na discussão de temas relacionados à influência dos jogos 
eletrônicos no comportamento social, afetivo e psicológico dos indivíduos. Destaca-se o trabalho desenvolvido por Abreu et al. (2008), cujo objetivo foi identificar a dependência da internet e dos jogos eletrônicos na população geral, apontando possível uso problemático dos jogos eletrônicos, principalmente entre jovens e adultos que desenvolveram transtornos psíquicos, como alteração do humor, ansiedade social, déficit de atenção, transtornos de personalidade, entre outros.

Já no eixo temático "Jogos Eletrônicos e Corporeidade" foram encontrados trabalhos que refletem, através da filosofia, sobre a relação entre os jogos eletrônicos e suas novas possibilidades de vivências corporais. Cruz Junior e Silva (2010) buscaram relacionar as conexões entre corpo, subjetividade e tecnologia, perpassadas pelos princípios da cibercultura. Para os autores a natureza do corpo digital é resultado daquilo que acomete o corpo real, exposto a tensões ou a traumas e os jogos eletrônicos nos dias atuais já não são apenas uma forma de distração, mas sim alternativa para se viver em sociedade, explorando a fantasia, o impossível, indo além do imaginário. A relação entre o sujeito e sua versão em um mundo virtual, não deve ser apenas vista como uma relação de mero consumo e vivência momentânea. O interesse dos internautas por essa forma de socialização demonstra a ideia de que o ciberespaço é um lugar propício para inventar e reinventar a própria vida e o próprio corpo. Outro trabalho nesse eixo foi o de Jensen; Capraro e Moraes e Silva (2017), no qual buscaram - amparados principalmente pelas análises sobre o corpo do intelectual francês David Le Breton - apresentar as significações sociais do corpo no mundo físico e as alterações com o surgimento do mundo virtual. Para os autores, mesmo o corpo tendo uma importância sociocultural, quanto maior a imersão no mundo digital, maiores são as chances desse mesmo corpo ser "dispensado e esquecido". O fato de o corpo estar 
fragmentando-se não significa que o indivíduo deixara de se relacionar com outros indivíduos, mas sim que surgirão novas formas - que são potencializadas pelo universo virtual - nas quais o sujeito pode metamorfosear-se e realizar ações que jamais faria na vida "real".

Por fim, o eixo "Jogos Eletrônicos e Esporte" caracterizou-se por trabalhos que analisam os jogos eletrônicos como manifestações esportivas, incluindo discussões sobre seu possível processo de esportivização e o surgimento da figura do atleta (ciberatletas). Para ilustrar esse eixo, destaca-se por exemplo o estudo de Macedo e Filho (2015), no qual apresentam o surgimento dos esportes eletrônicos (E-sports), analisando a gênese e a transformação de uma prática meramente de lazer para uma prática caracterizada como esportiva, inclusive com o surgimento de atletas, estabelecimento de regras específicas e campeonatos.

Após a apresentação dos eixos temáticos e seus respectivos trabalhos, mais uma vez percebe-se a diversidade teórica e interpretativa na qual o objeto foi tematizado. Suas análises versam desde questões relativas à influência dos jogos na transformação dos comportamentos dos indivíduos, perpassando questões que influenciam as novas possibilidades de lazer, bem como de consumo e trabalho, algo salutar para maiores aprofundamentos e interpretações sobre os jogos eletrônicos.

\section{Considerações Finais}

O presente artigo teve como objetivo apresentar algumas características da literatura científica sobre jogos eletrônicos, identificando as temáticas mais atendidas, os locais de publicação, os estratos dos periódicos publicados no sistema Qualis, bem como os autores e instituições com maior número de artigos publicados. Vale salientar 
que o estudo utilizou as principais bases de dados disponíveis para consulta, não se restringindo apenas a revistas indexadas apenas a plataformas que se traduzem em melhores estratos, o que pode auxiliar em uma análise mais ampla.

Como resultado, o estudo catalogou um número de 57 artigos publicados em 46 diferentes periódicos, sendo em 1999 o ano com a primeira publicação sobre a temática e o ano de 2016 com o maior número de trabalhos publicados. A maior quantidade foi registrada na RBCE e a na Psicopedagógica. A perspectiva da saúde apareceu como eixo temático de maior número de estudos publicados, seguidos de discussões sobre a relação dos jogos eletrônicos com a escola. Salienta-se ainda a diversidade epistemológica no trato do objeto na medida em que as produções se localizam em diferentes perspectivas e eixos teóricos, desde questões mais situadas nas ciências naturais, até aquelas amparadas em elementos das ciências humanas e sociais.

Quanto aos autores dos trabalhos, a grande maioria são doutores, e quanto as suas áreas de formação inicial, há leve predomínio de pesquisadores da área da Educação Física (29,07\%), seguidos de pesquisadores com formação em Psicologia (26,95\%), em Fisioterapia em terceiro, representando 10,63\% do total, já pesquisadores da área de Medicina, Nutrição e Pedagogia representam 6,38\%, 4,25\% e 4,25\% respetivamente.

Por fim, sugerem-se novos estudos que busquem analisar os jogos eletrônicos sob perspectivas científicas diversas, de modo a fornecer aos interessados no campo maiores indícios e elementos interpretativos desse fenômeno presente na contemporaneidade. 


\section{REFERÊNCIAS}

ABREU, C. N. et al. Dependência de Internet e de jogos eletrônicos: Uma revisão. Revista Brasileira de psiquiatria, v. 30, n. 2, p. 156-167, 2008.

AZEVEDO, T. Jogadores viram profissionais com a rede. Uol Jogos, São Paulo, 2004. Disponível em: http://www1.folha.uol.com.br/fsp/informat/fr1802200401.htm. Acesso em: 06 nov 2016.

BARACHO, A. F. de O; GRIPP, Fernando Joaquim; LIMA, Márcio Roberto de. Os exergames e a educação física escolar na cultura digital. Revista Brasileira de Ciências do Esporte, v. 34, n. 1, p. 111-126, 2012.

BALANCIERI, R. et al. A análise de redes de colaboração científica sob as novas tecnologias de informações e comunicação: um estudo na Plataforma Lattes. Ciência da Informação, Brasília, v.34, n.1, p.64-77, 2005.

CRUZ JUNIOR, G. ; SILVA, E. M. A (ciber)cultura corporal no contexto da rede: uma leitura sobre os jogos eletrônicos do século XXI. Revista Brasileira de Ciências do Esporte, v. 32, p. 89-104, 2010.

DE ALMEIDA REIS, L. J.; CAVICHIOLLI, R. F. Jogos eletrônicos e a busca da excitação. Movimento, v. 14, n. 3, p. 163, 2008.

FANTIN, M. Crianças e games na escola: entre paisagens e práticas. Revista Latinoamericana de Ciencias Sociales, Niñez y Juventud, 13 (1), pp. 195-208, (2015).

FERRARI, G. L. de M. et al. Associação entre equipamentos eletrônicos no quarto com tempo sedentário, atividade física e índice de massa corporal de crianças. J. Pediatria. (Rio J.), v. 91, n. 6, p. 574-582, 2015.

FIGUEIREDO, O; SBISSA, P. P. M. Efeito dos jogos eletrônicos sobre atenção seletiva. Ciência e Cognição, v. 18(2), p. 129-155, 2013.

GONÇALVES, Luiz Fernando et al. Mapeamento da produção do conhecimento sobre a modalidade do basquetebol nos periódicos brasileiros. Pensar a Prática, v. 20, n. 3, p. 461-475, jul./set. 2017.

JENSEN, L.; CAPRARO, A., M.; MORAES E SILVA, M. Jogos eletrônicos e corpo: as múltiplas identidades do indivíduo no espaço cibernético. Licere, v. 20, n.2, 2017.

LAZZAROTTI FILHO, A. et al. Modus operandi da produção científica da EF: uma análise das revistas e suas veiculações. Revista da Educação Física/UEM, Maringá, v. 23, p. 1-14, 2012.

LE BRETON, David. Antropologia do corpo e modernidade. 3. ed. Petrópolis: Vozes, 2013.

LÉVY, P. Cibercultura. São Paulo: Ed. 34, p. 11; 22; 251999. 
MACEDO, T.; FILHO, O.A. A Arena Espetáculo: quando as fronteiras de jogo e trabalho se rompem. Intercom, p. 1, 2015.

MOREIRA, T. S. ; MORAES E SILVA, Marcelo ; SOUZA, Doralice Lange ; MEZZADRI, F. M. . O perfil da produção científica em Língua Portuguesa sobre o Voleibol. Revista Motrivivência , v. 29, p. 119-135, 2017.

NEWZOO. A regional breakdown of the $\$ 99.6$ bn global games market (free report). Newzoo, 2016. Disponível em: https://newzoo.com/insights/markets/games/ . Acesso em: 06 nov 2016.

PERUCIA, A.; BALESTRIN, A.; VERSCHOORE, J. Coordenação das atividades produtivas na indústria brasileira de jogos eletrônicos: hierarquia, mercado ou aliança? Produção, v. 21, n.1, p. 64-75, 2011.

SIBILIA, P. O homem pós-orgânico: corpo, subjetividade e tecnologias digitais. In: Conexões. Relume Dumará, 2002.

\section{Endereço dos Autores:}

Heitor Luiz Furtado

Departamento de Educação Física

Universidade Federal do Paraná - Campus Jardim Botânico

Rua Coração de Maria, 92

Curitiba - PR - 80.210-132

Endereço Eletrônico: heitorluizfurtado@hotmail.com

Cahuane Corrêa

Departamento de Educação Física

Universidade Federal do Paraná - Campus Jardim Botânico

Rua Coração de Maria, 92

Curitiba - PR - 80.210-132

Endereço Eletrônico: cahuanecorrea@gmail.com

Michael Cani

Departamento de Educação Física

Universidade Federal do Paraná - Campus Jardim Botânico

Rua Coração de Maria, 92

Curitiba - PR - 80.210-132

Endereço Eletrônico: michaelcani2409@gmail.com

Patrick Joshuan Reitz Matendal

Departamento de Educação Física

Universidade Federal do Paraná - Campus Jardim Botânico

Rua Coração de Maria, 92

Curitiba - PR - 80.210-132

Endereço Eletrônico: joshuuan@hotmail.com 
Heitor Luiz Furtado, Cahuane Corrêa, Análise da Produção Científica sobre Jogos Eletrônicos...

Michael Cani, Patrick Joshuan Reitz Matendal e

Marcelo Moraes e Silva

Marcelo Moraes e Silva

Departamento de Educação Física

Universidade Federal do Paraná - Campus Jardim Botânico

Rua Coração de Maria, 92

Curitiba - PR - 80.210-132

Endereço Eletrônico: moraes_marc@yahoo.com.br 